\title{
Visualization for Planning and Simulation of Minimally Invasive Neurosurgical Procedures
}

\author{
L.M. Auer ${ }^{1}$, A. Radetzky², C. Wimmer ${ }^{1}$, G. Kleinszig ${ }^{1}$, F. Schroecker ${ }^{2}$, D.P. Auer ${ }^{4}$, \\ H.Delingette ${ }^{5}$, B.Davies ${ }^{3}$, and D.P. Pretschner ${ }^{2}$ \\ ${ }^{1}$ Institute of Applied Sciences in Medicine, ISM, Salzburg, Austria \\ ${ }^{2}$ Institute for Medical Informatics, Technical University of Braunschweig, Germany \\ ${ }^{3}$ Imperial College, London, GB \\ ${ }^{4}$ Max Planck Institute for Psychiatry, Munich, AG-NMR, Germany \\ ${ }^{5}$ INRIA, Sophia Antipolis, France
}

\begin{abstract}
A unit for training and simulation as well as for planning of minimally invasive operations of the brain is presented, called ROBO-SIM, for virtual patient positioning, planning of the surgical approach to a target in the depth, for anatomical orientation in the operating field and for manipulations of the virtual tissue. Methods are described for the visualization of the outer body surface (head), the virtual operating field in the depth of the brain, virtual surgical instruments and deformations (displacement, fragmentation) of the virtual tissue by simulated surgical manipulations. Mainly volume-rendered data from 3D-MRI are used. Surface views of the whole dataset are used for virtual patient positioning. For virtual endoscopic visualization of the small operating field within cavities of the brain (ventricles, cysts), a volume rendering application of "Volumizer" has been developed, called "Flight-Volumizer". Elastodynamic tissue deformation is achieved by a viscoelastic model on the surface of the cavities, which is simulated by neuro-fuzzy systems.
\end{abstract}

\section{Introduction}

With the advent of computer-assisted methods in surgery, image-guided planning has become an increasingly accepted procedure in neurosurgery under the term of "neuronavigation". As robotic manipulators are being considered to augment precision of microsurgical steps [1-3], complex preoperative planning and simulation is mandatory and will represent an important part of the total duration of a robot-assisted operative procedure. Therefore, the development of surgical simulators, comparable to flight simulators, has been initiated in a number of institutions. However, creation of the illusion of reality, which is most important for simulation and training of surgical manipulations, such as the real-time visualization of movements during manipulations, or the transfer of tactile sensations to the surgeon, or the visualization of the effect of robotic activities, have remained a formidable challenge for high-end graphical computing and other disciplines.

With the concept presented in this paper, the authors propose the use of a combination of volume- and surface-rendered data for visualization tools in a simulator for minimally invasive procedures. 3D-MRI datasets of the brains from actual patients are used to create views from the outer surface of the head (fig.1) as well as from inner surfaces such as the ventricular system or cystic brain lesions by aid of virtual endoscopy (fig.4) [4-6]. 


\section{Methods and Present State of Development}

The integral setup called ROBO-SIM is designed for manipulator-assisted virtual procedures through a trepanation in the skull of $1-2 \mathrm{~cm}$ diameter and a miniaturized approach of few millimeters diameter to target areas in the depth of the brain and its ventricular system. The present version of the system consists of a set of real instruments including a real neuro-endoscope mounted onto a passive manipulator arm with encoders and brakes (Impulse Engine, Immersion Corp., San Jose, USA) for the implementation of force-feedback and other constraints. In a more elaborate version as a planning station of the operating system ROBOSCOPE (project of the EU-Telematics programme), the system will include a robot arm, NEUROBOT, which is used by the surgeon as an active manipulator with inbuilt robotic capabilities such as active constraints, backtracking and a steady-distance tool. The surgeon who is planning and performing a virtual surgical procedure is thus working with the real instruments to be used for actual surgery, while looking onto a virtual scenario of the operating field, created by aid of a 3D-MRI-dataset of an actual patient.

For simulations, 3D-MRI-datasets from a 1,5T Signa echo-speed whole body scanner (GE Medical Systems, Milwaukee, USA) are used. The hardware platform for the development and use of the system is an Onyx2 Infinite Reality (Silicon Graphics, Mountain View, USA) as well as SGI O2-workstations and conventional PCs. The Onyx 2 is equipped with two R10000 processors (195 Mhz), $640 \mathrm{MB}$ main memory and $64 \mathrm{MB}$ texture memory. The graphics subsystem of the Onyx2 is a Infinite Reality2 graphics board with two channels, 4 graphics engines and one Raster Manager. The Onyx 2 is only needed for the real-time visualization and manipulation of the 3DMRI-dataset.

Figure 1 shows the graphical user interface and the functionality of ROBO-SIM. On the upper left corner, the virtual 3D viewer is demonstrated, where a view onto the patient's head is shown (Volume Rendering). The wheels and buttons at the sides of the viewer provide control functions, i.e. to rotate and trans-late the virtual camera. The three MR images at the bottom of the user interface show the orthogonal crosssections of the patients MR 3D-dataset. These images are peri-odically updated with the position of the virtual camera. A red marker in these images indicates the current camera position. The orientation of the virtual camera is represented by a red arrow, and a yellow arrow indicates the up-direction. A manipulator box gives the possibility to change the position and orientation of the camera. The figure shows the axial crossection with the manipulator to update the camera parameters. With the view selector button it is possible to switch to the transendoscopic view and to switch the ventricular surface model on or off. The position and orientation of the virtual endoscope, and in the "Endo" mode also the virtual camera is controlled by the Impulse Engine. The position of the camera in this mode is also displayed in the crossections and updated periodically. Different kinds of virtual instruments can be selected with the Toolselector-Menu. With the Toolmovement- Slider it is possible to control the instruments' length along the endoscope axis. The Window/Level-Sliders are used to adjust the threshold level for the 3D view. With the Inputfile-Textfield, different patient datasets can be loaded. If there is an existing surface model in addition to the volume data, it is possible to display this model in the reconstructed 3D-volume as indicated above. 


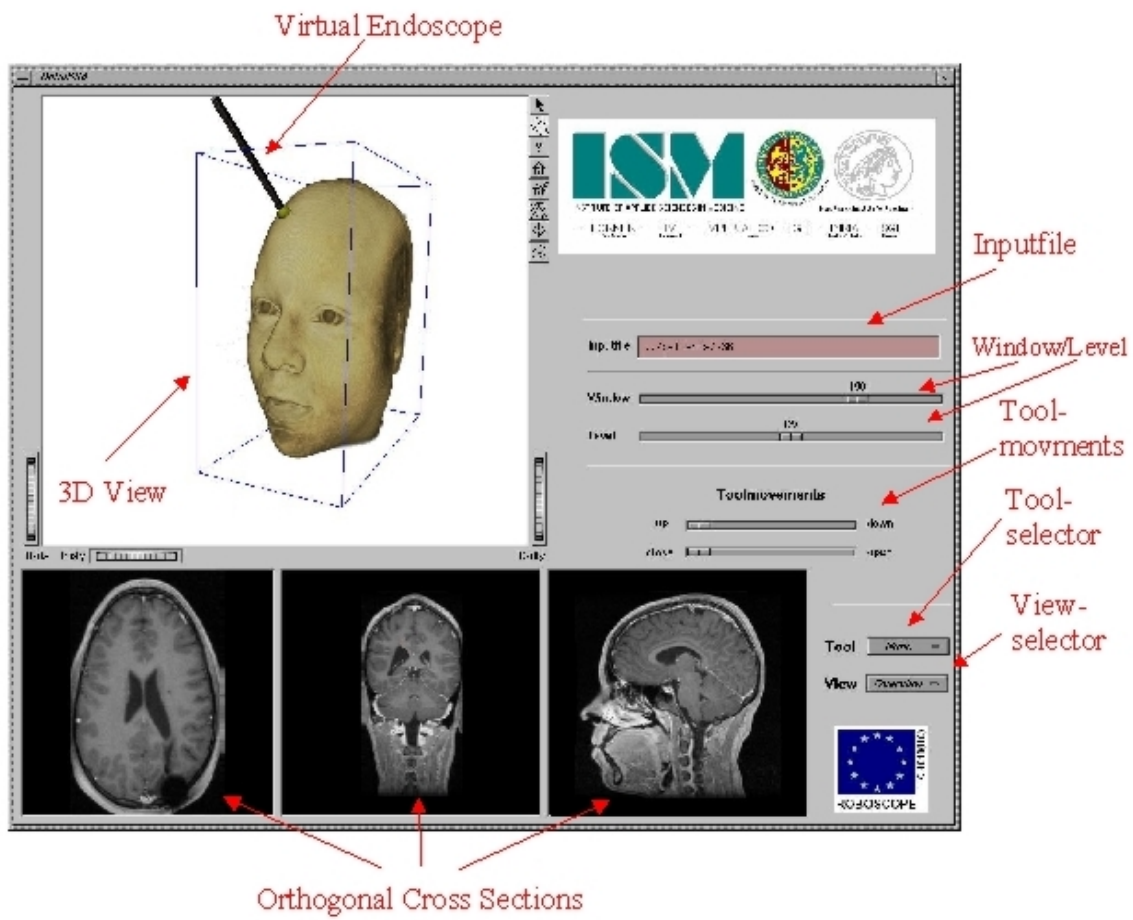

Fig. 1. The user interface of the simulator ROBO-SIM shows a view of the surface of the 3-DMRI-dataset as well as 3 planes of tomograms.

\subsection{Method of Virtual Endoscopy for Visualization of the Virtual Operating Field}

Before the development of software for virtual endocopy, various existing systems have been evaluated for their applicability in the frame of ROBO-SIM; detailed descriptions of this work are given elsewhere [7]. As a consequence of these analyses, a new software for virtual endoscopy was developed, called "Flight Volumizer", which uses the Advanced Programming Interface (API) OpenGL "Volumizer" [8] from Silicon Graphics as a basis. "Volumizer" is a volume rendering technique, a so-called PARC Algorithm (Polygon Aided Ray Casting), which uses tetrahedrons as volumetric primitives. This algorithm is highly accelerated for the efficient Infinite Reality Graphics hardware of the SGI Onyx2. 


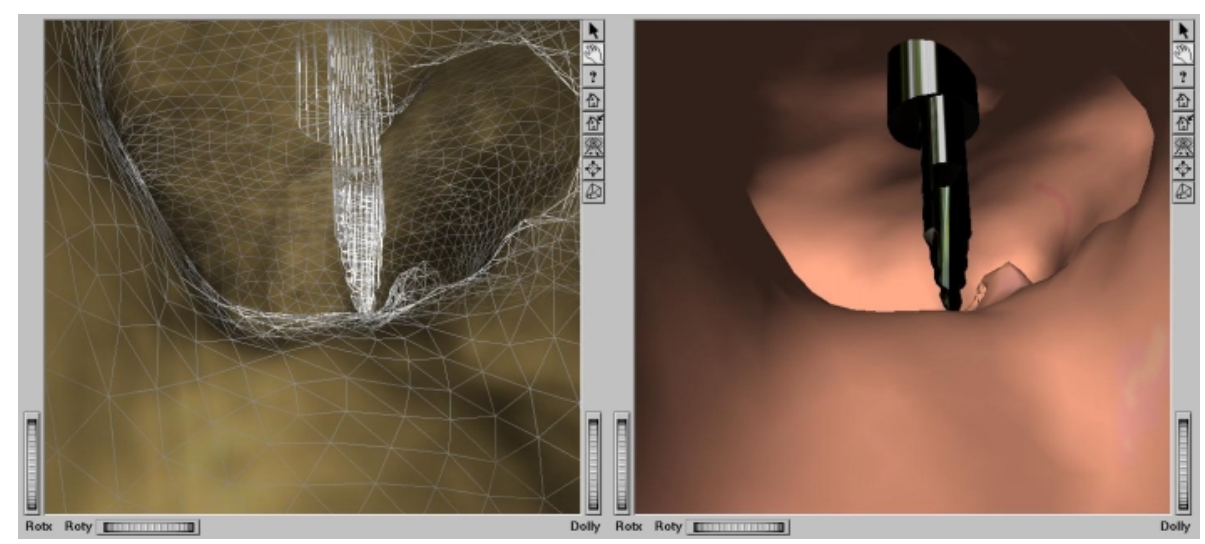

Fig. 2. Virtual endoscopic views of the lateral ventricle (left: surface-rendered image, right: view with Flight-Volumizer) with a virtual instrument applied transendoscopically. The right picture shows the overlay of the mesh underlying the surface rendering and the 3D dataset visualized with Volumizer. Each triangle on the surface is one face of a volumetric primitive at the same time.

Another advantage of "Volumizer" is the separation of the 3D dataset in Geometry (a set of volumetric primitives) and Appearance (voxel volume). Thus, Volumizer allows deformations and fragmentations by transforming the geometry without alterations of the voxel volume. Fragmentations are achieved by clipping the appearance on the geometry.

Besides, most of the voxel volume is not visible in virtual endoscopy because the usual view is transendoscopic. Therefore, only a small part of the $3 \mathrm{D}$ dataset has to be visualized, which is called the 'field of view'. The field of view is defined by the visibility limit of the virtual camera mounted onto the endoscope. In most cases this would be a cone or, for visualization on a monitor, a pyramid (the 'view-frustum').

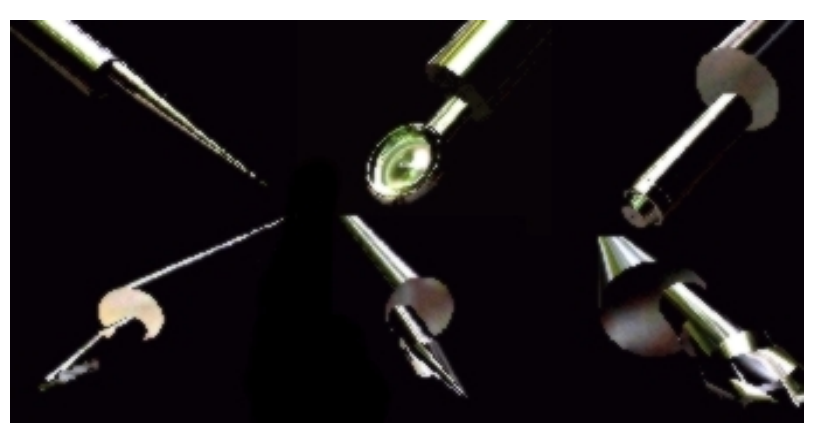

Fig. 3. Set of virtual instruments, which are introduced through the endoscope (scissors, biopsy forceps, grasping forceps, curette, needle, suction tube). to be applied to the tetrahedrons inside the view frustum and thus simplifying the frustum is located in the camera, and its basis is perpendicular to the camera's objective. Only the volumetric primitives inside the view frustum are in the field of view, and only these tetrahedrons have to be shown. Thus, by disabling all other tetrahedrons, it is possible to increase the rendering speed considerably.

Furthermore, virtual deformations only have The tip of the view- 
required algorithms (see section 2.4.2). The software is developed using $\mathrm{C}++$ and the object oriented 3D-toolkit Open Inventor [11], where Volumizer's functionality is fully integrated.

Besides visualization of ventricular surfaces of the volume-rendered dataset, a surface rendered model of the ventricular surface, coregistered with the volume-rendered dataset, may be used for visualization; alternatively, a combination of both can be used. The surface models were generated as a preprocessing step by aid of a semiautomatic segmentation tool [9]. The resulting surface model was integrated in the virtual environment (OpenInventor SceneGraph) in addition to the volume dataset. The transparency of certain intensities can be set with the Window/Level-sliders (see fig.1).

The view shown in figure 2 is intentionally different from a transendoscopic view in order to provide a better overview of the situation from a technical standpoint. Primarily, however, visualization of the operating field for virtual surgery would be transendoscopic. Therefore, the number of volumetric primitives inside the viewfrustum would be much less, because the endoscope is directly facing the ventricle's surface. Using a transendoscopic view, interactive frame rates of approximately 10 frames per second are possible with a volume-rendered dataset. The virtual instruments available for selection by the surgeon are shown on fig.3.

\subsection{Visualization of Virtual Patient Positioning and Approach Planning}

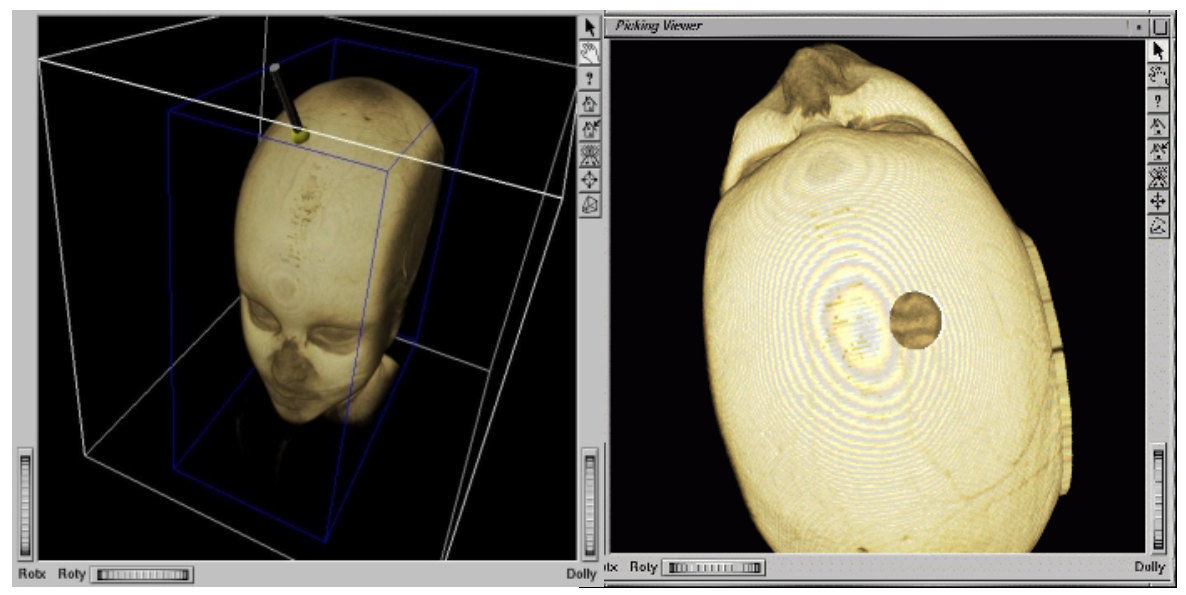

Fig. 4. Following positioning of the virtual patient, a port of entry into the skull (trepanation) is selected by aid of the "virtual craniotomy" tool, which allows to view the cerebral cortex for decisionmaking.

For virtual positioning of the patient's head, it is possible to translate and rotate the dataset with respect to the virtual endoscope using a manipulator box, which surrounds the entire head and can be freely translated and rotated. For the simulation of a trepanation, the virtual burrhole is placed and adjusted to the underlying anatomy of the brain surface (fig.4). This virtual burrhole is defined by a cylinder with a height 
of $8 \mathrm{~mm}$ along the negative gradient of the surface. The volume around the cylinder is filled with a geometry of tetrahedrons, which replaces the standard geometry consisting of a tetrahedrated cube containing the whole dataset. As a result, the entire head exept the skullbone with a selected radius at the desired position is visualized (fig.4).

As a next step in the virtual planning procedure, the target point for arrival of the instrument in the depth of the operating field may be selected by respective placement of the tip of the virtual instrument. The viewing direction inside the dataset can be freely chosen to allow e.g. virtual transendoscopic visualization or a view onto the tip of the instrument in relation to the surrounding anatomical structures (fig.5).

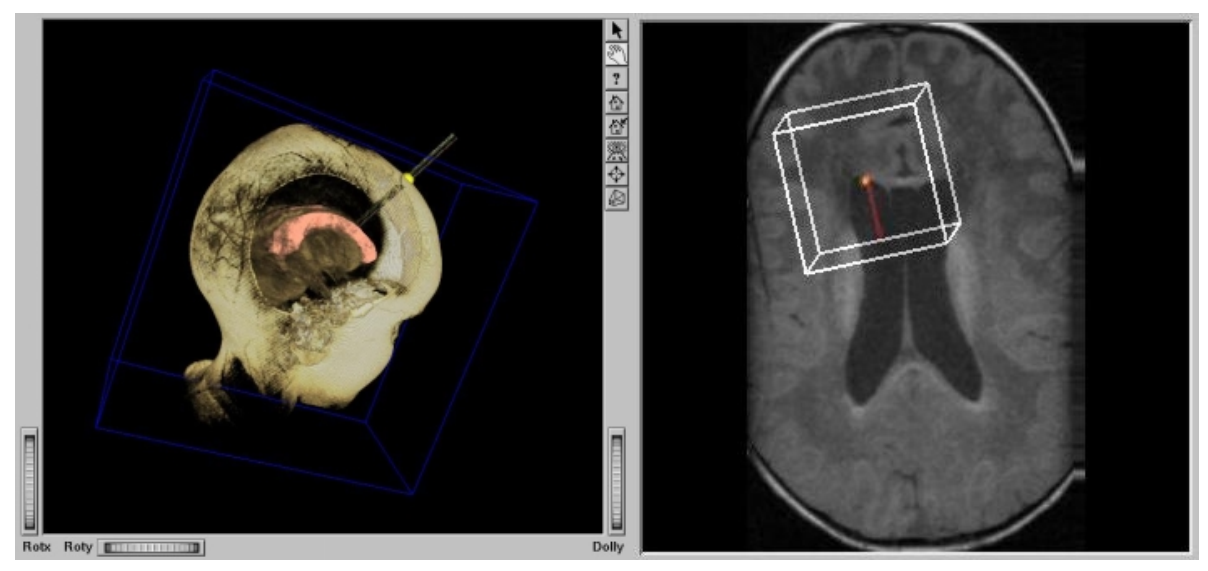

Fig. 5. Planning of the target point is possible by placing the tip of the instrument such as the virtual endoscope (left) or by selecting a point in the tomograms, using the respective selector box (right). The figure on the left is an overlay of a semi-translucent volume-image and the patient's surface-rendered ventricular system.

\subsection{Elastodynamic Tissue Deformation}

To simulate deformable tissues, a dynamic component is required. Every tissue has an assigned mass, elasticity and viscosity. The appearance or behavior of any deformation depends on these parameters to a great par ${ }^{1}$. To describe these physical conditions, a viscoelastic model can be used [16].

One of the first applications of viscoelastic models for the simulation of elastic deformable models was developed by Terzopoulos et al. [14]. Some improved techniques were presented, for example, in [15-18]. One of the main problems of these approaches is the difficulty to derive the parameters of the physical model - thus leading to an insufficient biological realism [17] - and the high computational demands during simulation. Furthermore, some of these approaches can not be used if

${ }^{1}$ Further parameters (depending on the used model structure) are, for example, friction and fragility. These parameters are not considered in the following, but they can be defined by the presented approach, too. 
cuts have to be performed. To resolve these problems, a neural network architecture [19] was developed, which is able to simulate viscoelastic models [21]. In this way it is possible to learn the parameters of the physical model and to speed up the simulation by use of problem specific propagation ${ }^{2}$ procedures. Furthermore, a fuzzy system was implemented to initialize the network parameters if some prior knowledge about the model, like stiffness, elasticity or shiftability, is available [12, 21].

Viscoelastic Models. In viscoelastic models [16] the whole mass of the elastic objects (e.g. virtual organs) is divided up between the mass points of the model, which are represented as nodes in a mesh. Every node of the mesh can be connected elastically or viscously to its neighbors. The connections are represented as viscoelastic elements. By use of this model, deformations, transections, coagulations etc. can be performed virtually. This is done by applying external forces to the nodes of the mesh, which are affected by collisions with virtual instruments. Also, the displacement of a node can be given and the resulting forces can be calculated.

Viscoelastic models of deformable objects can be simulated 'as a whole', which is comparable to the computation of conventional differential equation systems. This is called total deformation [20] and can be used especially if the objects are not fixed or connected with other objects. However, the total deformation can only be used in real time with small objects consisting of only a few hundred nodes, whereas the complex surface of the ventricle shown in fig. 2 has about 20000 nodes. However, for a realistic impression only deformations within the view-frustum have to be simulated. This can be done with local deformation [20]. A local deformation is the same as a total deformation except that the local deformation is limited to the part of the tissue, which is directly influenced by the contact with an instrument. Using traditional deformation models, this leads to insufficient realism because local deformation is a heavy simplification of the real dynamic system. Another approach uses neuro-fuzzy systems [22] for the simulation of viscoelastic models [21]. With neuro-fuzzy systems, the deformation behavior can be learned or given by simple linguistic terms regardless of the simplification done by local deformation. Not the exact physical model but the visual realism is imitated. For surgical simulation, it is sufficient if a virtual deformation appears real to an expert surgeon.

A comprehensive introduction of the simulation with neuro-fuzzy systems is given in $[13,21]$.

Virtual Deformations and Fragmentations. In general, virtual deformations of 3D datasets can be simulated by transforming the volumetric primitives of Volumizer's geometry. However, the geometry has to be connected with a viscoelastic model in such a way as to fill the whole visible dataset without intersections of volumetric primitives. One approach is to create viscoelastic models, which are inspired by crystal structures. In [10] an algorithm is presented, which can be used to fill a diamond crystal structure with volumetric primitives. Diamond crystals, represented as viscoelastic models, have the opportunity that large volumes can be filled with comparatively few springs and nodes. By connecting the viscoelastic model with the

${ }^{2}$ Propagation is the computation process of neural networks. In this case it is similar to algorithms for solving systems of differential equations [13]. 
volumetric primitives, deformations of 3D datasets can be simulated simply by deforming the viscoelastic model.

However, simulating deformations of the whole volume is not necessary if an additional surface model exists. For example, the segmented surface of the ventricle shown in fig. 2 can be used to create a geometry of tetrahedrons starting from the ventricle's surface in direction of the brain surface and the skull. Each triangle of the surface defines the base of a prism, which can be described by three tetrahedrons. The surface itself is defined by a viscoelastic model, whose visualization is optional. In case of a collision with a virtual instrument, the surface is deformed using local deformation (see fig. 6). This causes a transformation of the prisms and thus a deformation of the $3 \mathrm{D}$ dataset. The generation of the prisms is very fast so that they can be created within the view-frustum during visualization. Thus, only the visible part of the volume has to be shown and deformed.
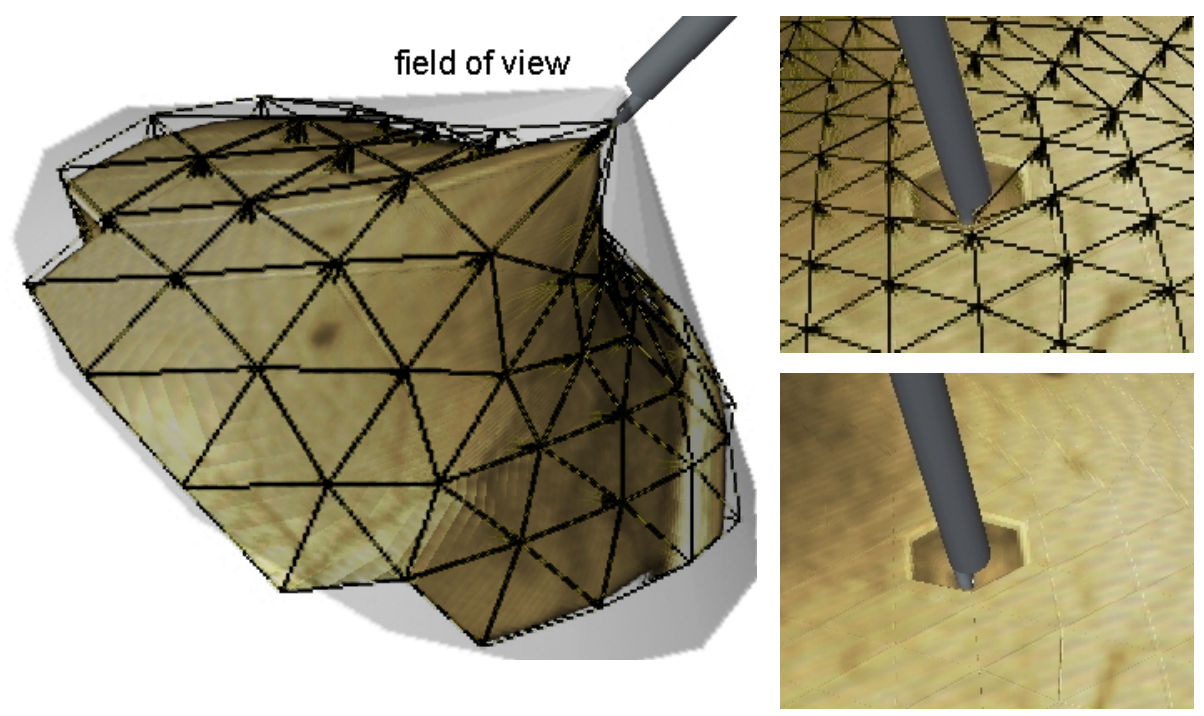

Fig. 6. Virtual deformations and fragmentations of a MR-dataset by use of an endoscope. Only the tetrahedrons inside the view-frustum are shown (left). On the right side a fragmentation is done by clipping on Volumizer's geometry.

Another advantage of this approach is the possibility for an easy implementation of fragmentations, e.g. virtual removal of tissue by aid of a biopsy forceps. By changing the mapping between the geometry and the appearance (cp. section 2.2) the voxel volume is clipped instead of deformed (see fig. 6). At the first contact with the forceps, the volume is deformed until the forceps is closed. Then, the deformation of the volume at the collision points remains permanent, which can be done by relaxing the involved springs. The next step is to change the mapping of geometry and appearance to clip the volume at this location. The fragmented volume remains in the dataset, but it is excluded from rendering by the clipping function of Volumizer. 


\section{Discussion}

Simulation of microsurgical manipulations and their visualization has become technically possible on a widely acceptable financial level of graphic computing. In several more years, hardware costs for the same computing power will be substantially reduced, or significantly more possibilities will be available for the same amount of money.

Several important factors of surgical simulation can already be dealt with by newest technical development such as visualization of the virtual operating field of an actual patient's anatomy and of elastodynamic tissue properties, propagation of some force feedback to the surgeon, manipulation with real instruments while seeing virtual instruments in the virtual operating field similar to the aspect in real surgery.

The simulation of bleeding, considered as an important factor for simulation of surgery in other body regions, is a minor factor in minimally invasive neurosurgery for the following reason: one of the basic strategies of surgical manipulations through minimally invasive approaches is preventive hemostasis by coagulation; this means that tissue is coagulated before it is removed, whenever a disturbing extent of hemorrhage can not be excluded with very high probability.

Typical colors of classical landmarks for endoscopic neurosurgery will be visualized by aid of a deformable surface model of these structures supplied with surface structure and color from a typical example of real endoscopic microsurgery.

More detailed force feedbacks and tactile sensations will not create a major progress for minimally invasive neurosurgery, because very little tactile information is available for the surgeon in real surgery, caused by friction-resistance during transendoscopic application of microinstruments. Individual mechanical tissue properties may be preselected with the present state of development; additional information will be imported by aid of a model database for a number of pathologies. However, the same as the individual color of patient's tissues as mentioned below, simulation of the mechanical properties of single voxels of an individual patient's tissue will remain a considerable technical challenge.

However, a number of other important factors still represent a major technical problem and thus a formidable challenge: one is the basic color of an individual patient's brain tissue, and more so, the individual color of pathology such as an individual tumor. Here, the solution might be to create a database of typical examples from real surgery, at least from the surface texture of a pathological tissue (individual colors of volumes of interest as such as the interior of tumor will remain very complicated).

Yet another factor which plays an important role in creating the illusion of a real situation during virtual surgery is the behavior of intracranial tissue compartments as a consequence of changes in intracranial pressure as well as of intracranial volume compartments: Changes in pressure of the irrigation fluid causes characteristic floating movements of the septum pellucidum and other thinwalled structures. Moreover, removal of larger amounts of intracranial volume such as cerebralspinal fluid or cyst fluid or tumor cause a readaptation of the remaining tissue compartments according to physical conditions in the closed system of a cranial cavity. Thus, physiological parameters such as the intracranial pressure - volume relationship will remain a challenge for the creation of a perfectly simulated surgical scenario, realistic enough to allow the use of such device for comprehensive training of real surgery. 
In summary, promising progress has been made in the creation of virtual worlds for a simulator of minimally invasive neurosurgery, however, several problems such as individual physiological factors will remain a formidable technical challenge.

\section{Acknowlegements}

This project is supported by the Austrian Ministery of Research (grant to ISM) and by the European Commission, programme telematics (nr. 4.018, Coordinator Dr.V.Paul, IBMT, Fraunhofer Institut für Biomed. Technik, AG Medizin-Telematik, St.Ingbert, D).

The consortium includes, besides the institutions of the authors and IBMT, Katholiek Univ. Leuven, Belgium, Silicon Graphics Inc., Cortaillod, Switzerland, FokkerControls, Amsterdam, The Netherlands, Kretz-Technik, Zipf, Austria, and a consortium of clinical validation centers from the departments of Neurosurgery of the Universities of Greifswald, Germany, Ghent, Belgium, Copenhagen, Denmark, Creteil/Paris, France, Nijmegen, The Netherlands).

\section{References}

1. Benabid-AL; Cinquin-P; Lavalle-S; Le-Bas-JF; Demongeot-J; De-Rougemont-J. Computer-driven robot for stereotactic surgery connected to CT scan and magnetic resonance imaging. Technological design and preliminary results. APPL-NEUROPHYSIOL. 50/1-6 (153-154) 1987

2. Davies, B.L., Ng, W.S., Hibberd, R.D., "Prostatic Resection; an example of safe robotic surgery." Robotica, Cambridge University Press, Vol 11, pp561-566, 1993.

3. Goradia, T.M., R Taylor, Auer LM. Robot-Assisted Minimally Invasive Neurosurgical Procedures: First Experimental Experience. Lecture Notes in Computer Science 1205, Springer 1997, pp319-22

4. Auer LM, Auer D.P., JF Knoplioch. Virtual Endoscopy for Planning and Simulation of Minimally Invasive Neurosurgery. Lecture Notes in Computer Science 1205, SpringerVerlag 1997, pp 315-18

5. Auer D.P., Auer LM. Virtual Endoscopy. A new Tool for Teaching and Training in Neuroimaging. Int.J. of Neuroradiol. 4, 1998, 3-14.

6. Auer, L.M., Auer, D.P. Virtual Endoscopy for Planning and Simulation of Minimally Invasive Neurosurgery. Neurosurgery 43, 1998, 529-548.

7. Kleinszig, G., Auer, D.P., Auer, L.M., Virtuelle Neuro-Endoskopie. Proc. OGBMT, Vienna 1998.

8. Grzeszczuk R, Henn C, Yagel R. Advanced Geometric Techniques for Ray Casting Volumes. In: Course Notes, 4, SIGGRAPH'98, 1998.

9. Delingette, H., General Object Reconstruction based on Simplex Meshes, INRIA Techn. Report No 3111, Feb. 1997

10. Radetzky, A., Wimmer, C., Kleinszig' G., Brukner, M., Auer, L.M., Pretschner, D.P. Interactive Deformable Volume Graphics in Surgical Simulation. In: International Workshop on Volume Graphics, 1999. 
11. Wernecke J. The Inventor Mentor. New York: Addison-Wesley, 1994.

12. Radetzky A, Nürnberger A, Pretschner DP. Elastodynamic Shape Modeler: A Tool to Define the Deformation Behavior of Virtual Tissues. In: RadioGraphics, 2000; 20(3) (to appear)

13. Nürnberger A, Radetzky A, Kruse R. A Problem Specific Recurrent Neural Network for the Description and Simulation of Dynamic Spring Models. Proc of the Int Joint Conference on Neural Networks, 1998; 468-473.

14. Terzopoulos D, Platt J, Barr A, Fleischer, K. Elastically deformable models, In: Computer Graphics, 1987; 21(4): 205-214.

15. Terzopoulos D, Fleischer K. Deformable Models, The Visual Computer, 1988; 4:306-331.

16. Terzopoulos D, Fleischer K. Modeling inelastic deformation: Viscoelasticity, plasticity, fracture. In: Computer Graphics, 1988; 22(4):269-278.

17. Cotin C, Delingette H, Ayache N. Efficient Linear Elastic Models of Soft Tissues for realtime surgery simulation, INRIA report no. 3510, INRIA Sophia Antipolis, 1998.

18. Cotin C, Delingette H, Ayache N. Real-time elastic deformations of soft tissues for surgery simulation, INRIA report no. 3511, INRIA Sophia Antipolis, 1998.

19. Rojas R. Neural Networks - A Systematic Introduction, Berlin: Springer, 1996.

20. Radetzky A, Nürnberger A, Teistler M, Pretschner DP. Elastodynamic shape modeling in virtual medicine. In: Proc. of Int Conference on Shape Modeling and Applications, IEEE Computer society, 1999; 172-178.

21. Radetzky A, Nürnberger A, Pretschner DP. Simulation of elastic tissues in virtual medicine using neuro-fuzzy systems, Kim, Y.; Mun, S.K. (ed), In: Medical Imaging 1998: Image Display. Proc. of SPIE Vol. 3335, 1998; 399-409.

22. Nauck D, Klawonn F, Kruse R. Foundations of Neuro-Fuzzy Systems, New York: John Wiley \& Sons Inc., 1997. 